\title{
Revascularization of Skeletal Muscle Transplanted into the Hamster Cheek Pouch: Intravital and Light Microscopy
}

\author{
John A. Faulkner, Stefani W. Weiss, and John K. McGeachie \\ Department of Physiology, The University of Michigan Medical School, \\ Ann Arbor. Michigan 48109
}

Received August, 1982

\begin{abstract}
Small fiber bundles from the extensor digitorum longus (EDL) muscle were transplanted into the hamster cheek pouch to observe the process of revascularization. We tested the hypothesis that blood vessels of a graft degenerate and that revascularization results from the ingrowth of blood vessels from the cheek pouch into the graft. Degeneration and regeneration of muscle fibers followed a pattern similar to that reported for autografts of whole muscles in rats. Blood vessels in the graft that were adjacent to vascularized host tissue survived. At 1 day, surviving blood vessels in the graft had normal structure but erythrocytes were packed tightly in the lumen. At 2 days, sprouts from surviving graft vessels had grown into the cheek pouch. Between 2 and 2.5 days, anastomoses of blood vessels from the graft with those of the cheek pouch reestablished circulation. After circulation was established, the blood vessels contained well-spaced erythrocytes. By 3 days, blood vessels which increased in number and diameter throughout the graft occupied $40-60 \%$ of the graft. Over the next $24 \mathrm{hr}$, blood vessels in the graft regressed toward the control value. We conclude that blood vessels in muscle grafts in the cheek pouch survive transplantation, and that circulation in grafts is reestablished by the anastomoses of blood vessel sprouts from the graft with the blood vessels of the host.
\end{abstract}

\section{INTRODUCTION}

Free whole muscles have been transplanted successfully into a variety of sites in rats (Carlson and Gutmann, 1975), cats (Faulkner et al., 1980), and monkeys (Markley et al., 1978). The proximal and distal tendons were sutured to the tendon stumps, but revascularization and reinnervation occurred spontancously (Carlson and Faulkner, 1983). The temporary ischemia following transplantation resulted in necrosis of all but a few peripheral skeletal muscle fibers, which survived by diffusion of oxygen and nutrients from adjacent blood vessels (Carlson and Faulkner, 1983). These surviving fibers may be destroyed by bupivacaine, a muscle anesthetic (Carlson, 1976). Following degeneration of the necrotic fibers, myogenic cells, which survived the ischemic period, formed new functionally normal muscle fibers (Carlson and Faulkner, 1983).

The ischemia of transplantation also caused degeneration of blood vessels (Clark and Blomfield, 1945; Vracko and Benditt, 1972; Hansen-Smith et al., 1980). Most cell components, except the basal laminae, underwent intrinsic and cell-mediated destruction (Hurley and Edwards, 1969; Vracko and Benditt, 1972; 
Hansen-Smith et al., 1980). The revascularization of whole-muscle grafts has been described as a process by which blood vessels grow into the ischemic graft from the host tissue (Hansen-Smith et al., 1980) since it is not clear that any of the cellular elements of the vasculature survive transplantation.

The cheek pouch of the hamster has becn used as a transplantation site for tumors (Greenblatt and Shubik, 1968), and cardiac muscle cells (Greenblatt et al., 1971; Cornish et al., 1979), but not previously for skeletal muscle grafts. Use of the cheek pouch site and cheek pouch chamber allowed serial observation with intravital microscopy. Our purpose was to transplant small bundles of approximately 20 skeletal muscle fibers, previously treated with bupivacaine, into the hamster cheek pouch to observe the process of revascularization. We hypothesized that: (1) The blood vessels in the graft would degenerate, and (2) revascularization would occur through growth of new blood vessels from the blood vessels of the cheek pouch into the ischemic graft.

\section{MATERIALS AND METHODS}

Female golden hamsters (Mesocricetus auratus), body mass 115 to $135 \mathrm{~g}$, were anesthetized with an intraperitoneal injection of sodium pentobarbital $(100 \mathrm{mg} /$ $\mathrm{kg}$, ip). One extensor digitorum longus (EDL) muscle was removed. Four to six small bundles, each consisting of approximately 20 intact skeletal muscle fibers, were dissected from the EDL muscle using a dissection microscope and microsurgical techniques. The bundles were immersed in bupivacaine for $10 \mathrm{~min}$ and then washed three times with a mammalian Krebs-Ringer solution (Close, 1965).

The transplantation procedure was a modification of that used by Cornish et al. (1979) to transplant cardiac fibers into the cheek pouch. The cheek pouch of the hamster was cleaned with a saline-soaked gauze pad and then wiped with Betadine solution. The baseplate of the cheek pouch chamber was attached to a spring-loaded holder which was inserted into the cheek pouch (Greenblatt et al., 1969). About the middle of the cheek pouch, an incision was made through the skin over the site of the baseplate. The nonvascular mucoareolar membrane was removed avoiding damage to blood vessels on the surface of the cheek pouch. Three grafts were sutured into each cheek pouch with 10-0 suture. The sealed cap of the cheek pouch chamber was fixed to the baseplate. The skin was pulled around the chamber and fixed securely with a purse-string suture.

Grafts were observed at $0.5,1.0,1.5,2.0,2.5,3.0,3.5,4.0,5.0$, and 14 days after transplantation. The hamsters were anesthetized as described previously. The cheek pouch chamber was attached to a holder that facilitated viewing by intravital microscopy. Transillumination was obtained by placing a light tube in the cheek pouch and each graft was photographed at low power. This provided an overview of the structure and vasculature of the graft. At each time period, the total vasculature of each graft was explored carefully for any sign of blood flow. When blood flow was observed, red blood cell velocity was measured in larger vessels using a red blood cell velocity correlator and in smaller vessels by a frame-by-frame analysis of video tapes (LaLone and Johnson, 1978). Vessels in which flow was measured were identified by landmarks so that the same vessel could be observed in $1-\mu \mathrm{m}$ sections. 
Following intravital microscopy, the grafts were fixed in situ for $30 \mathrm{~min}$ by filling the cheek pouch chamber with $4 \%$ cacodylate-buffered glutaraldehyde $(\mathrm{pH}$ $=7.2$ ). To facilitate correlation of observations made with intravital microscopy and those of fixed sections, a cut was made adjacent to a segment in which vessels had been inspccted by intravital microscopy and a marker was cut on the opposite side. The 1-mm-long segments of grafts and the attached cheek pouch were removed and fixed in fresh glutaraldehyde. As controls, entire EDL muscles were removed, cut into $1-\mathrm{mm}$ cubes, and fixed in $4 \%$ glutaraldehyde. After washing in buffer, samples were postfixed at room temperature with cacodylate-buffered $1 \%$ osmium tetroxide to which $40.2 \mathrm{mg}$ of sucrose had been added. Tissues were rinsed in distilled water and dehydrated in an alcohol series. Propylene oxide was used as a transitional solvent before embedding in Polybed 812. Care was taken to orient the samples in flat capsules. One-micrometer sections were made and stained in toluidine blue. At least one segment of each of the grafts obtained between 0.5 and 2.5 days was serially sectioned by taking one section, skipping five sections, and then taking another section. All segments of one 2-day graft were sectioned in this way.

\section{RESULTS}

Each of the skeletal muscle fibers in the graft degenerated and regenerated during the 14-day period of observation. The blood vessels in the graft and in the cheek pouch underwent major changes that resulted in the successful revascularization of the graft.

\section{Skeletal Muscle Fibers}

In $1-\mu \mathrm{m}$ sections, skeletil muscle fibers showed varying stages of degeneration and later regeneration during the 14-day period of observation. At 0.5 -days, some fibers showed vacuolation of the sarcoplasm (Fig. 1). Other fibers had lost their striations and the myofibrils had begun to fragment. Gradations between the stages of degeneration were observed within single as well as among different fibers (Fig. 2). Some fibers, usually 1 or 2 , of the approximately 20 fibers in the graft, appeared almost normal during the first days. These fibers had mean crosssectional arcas of approximately $2000 \mu \mathrm{m}^{2}$ which was not significantly different from control values. No significant changes in fiber morphology were observed between 0.5 and 1.5 days, but invasion by inflammatory cells occurred by 1.5 days. Inflammatory cells were most noticeable in fibers located along the graftcheek pouch border (Fig. 3).

By 2 days, degeneration of muscle fibers was more advanced. Some fibers were severely vacuolated and others showed varying degrees of myofibrillar degeneration (Fig. 4). From 2 to 2.5 days, there was increased breakdown and phagocytic infiltration of individual muscle fibers. The fibers nearest the cheek pouch were heavily invaded by macrophages especially in areas adjacent to cheek pouch blood vessels. Fibers on the upper surface of the graft were less affected, with vacuolation, myofibrillar degeneration, and edema, but no phagocytic infiltration. Most muscle fibers maintained their original outline and shape. Many mitotic figures were seen in association with the outer surface of muscle fibers (Fig. 5). 


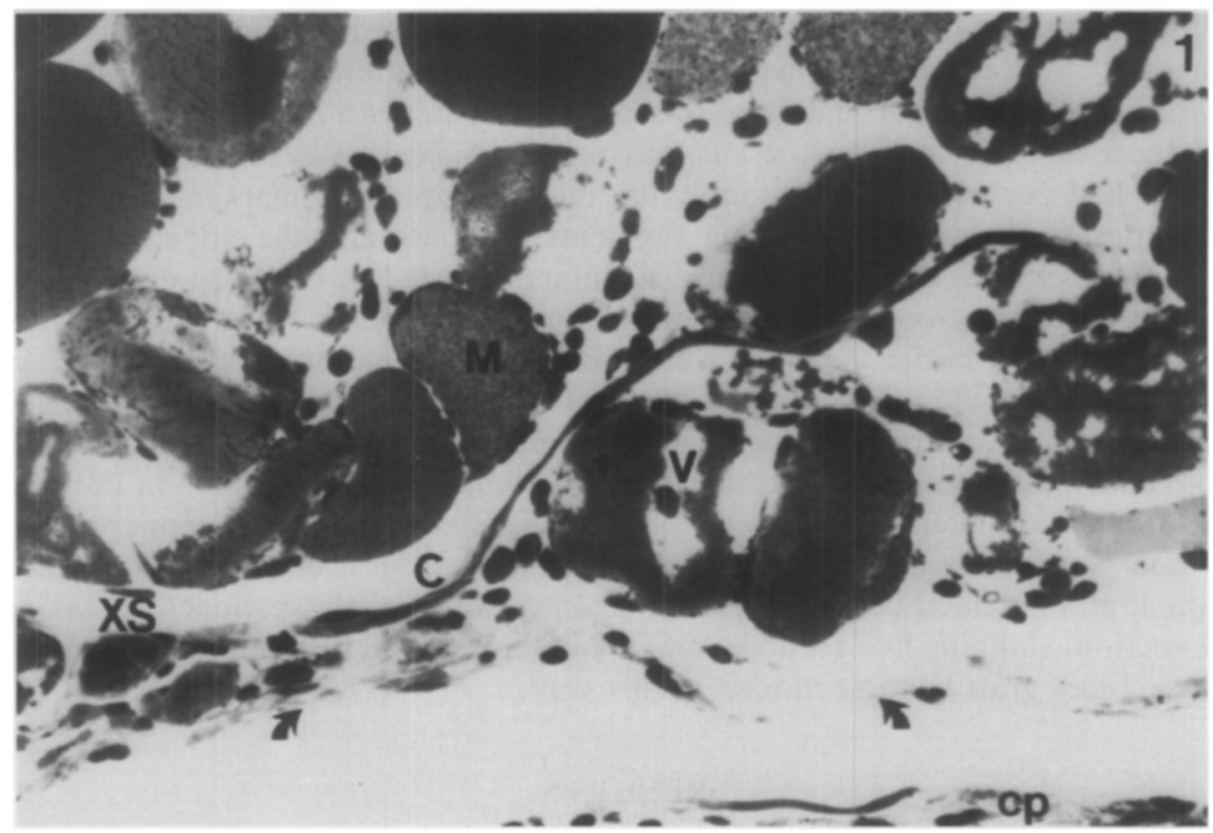

FIG. 1. Muscle fibers in this $\mathbf{0 . 5}$-day graft are at various stages of degeneration. Some are severely vacuolated $(\mathrm{V})$, whereas others are more normal in appearance $(\mathrm{N})$. A cross section of a capillary (XS) and longitudinal branch (C) which is $0.25 \mathrm{~mm}$ long are located in the graft. The curved arrows point to the graft border below which is the gap between the graft and the cheek pouch. At 0.5 day this gap has not been infiltrated by inflammatory cells and thus appears clear. The cheek pouch (cp) is barely visible in the lower right-hand corner of the picture. Magnification: $\times 575$.

By 3 days, some muscle fiber profiles were no longer recognizable (Fig. 6). Mitotic figures in the outer regions of the muscle fibers were even more evident than at earlier times. By 3.5 days, almost all evidence of the original muscle fibers had disappeared, apart from the presence of an occasional fiber which was darkly staining and morphologically intact. The graft area was one of intense cellular infiltration with macrophages and cells presumed to be fibroblasts and myoblasts. There were a few small myotubes (Fig. 7).

Myotube formation was well advanced by 4 days (Fig. 8). Mitotic and macrophagic activity had declined compared to that of 2.5 to 3.5 days. Collagen formed a substantial, but varying proportion of the graft area. Intravital microscopy of grafts at 5 days, showed spontaneous, rhythmical contractions of skeletal muscle fibers. Light microscopic observation indicated that myotube formation was well advanced (Fig. 9). A few small myofibers, with peripheral nuclei, were scattered

FIG. 3. At 1.5 days some of the muscle fibers are infiltrated by phagocytic cells (star), especially if they are located near the graft-cheek pouch border (the bottom of the graft is marked by curved arrows). The amount of vacuolation and degeneration of musclc varies between samples and within any sample. The fibers in this section have undergone less degeneration than is typical. A typical graft vessel (C) contains packed erythrocytes, and a typical cheek pouch (cp) blood vessel (B) contains well-spaced erythrocytes. The gap between the graft and cheek pouch can no longer be easily distinguished. Magnification: $\times 425$. 


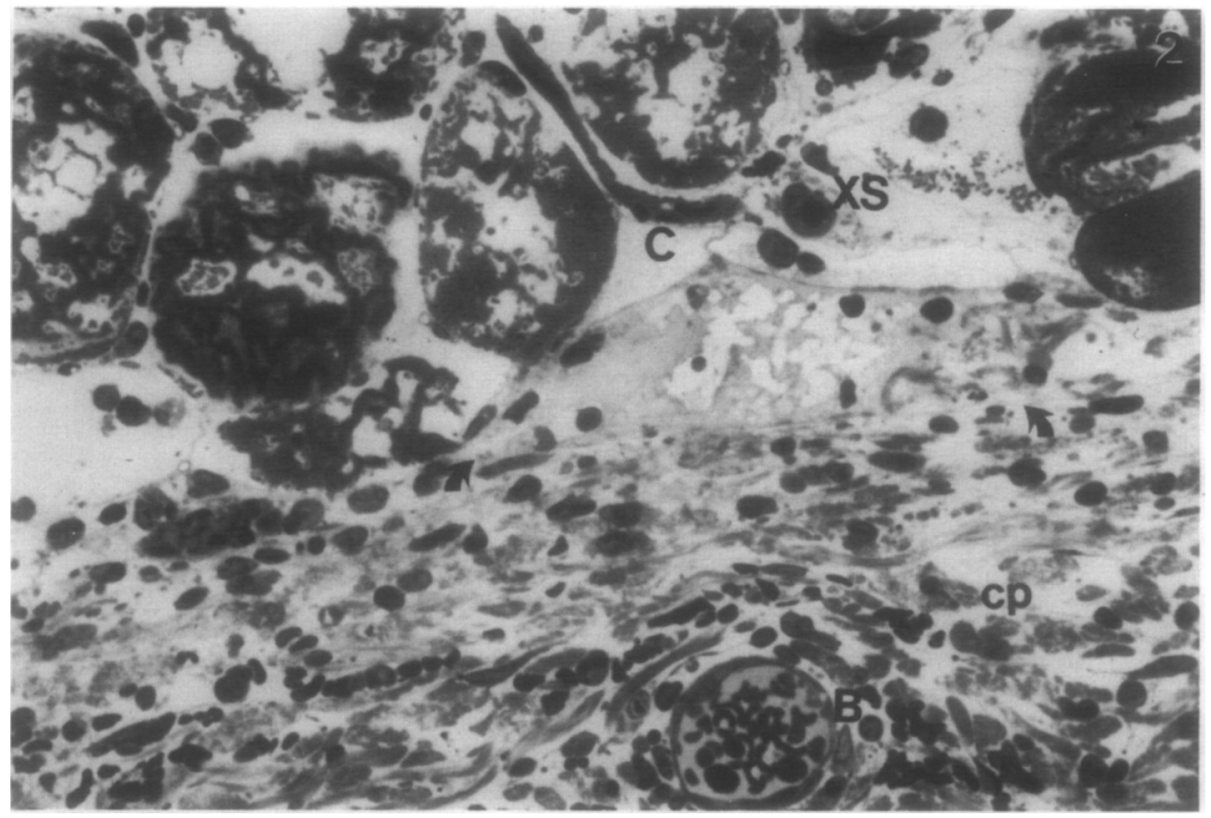

Fig. 2. In this 1-day graft the fibers are similar to those seen at 0.5 days, except in this particular section the fibers are extremely vacuolated. The amount of vacuolation varies from section to section. A cross section of a typical graft blood vessel (XS) and one of its longitudinal branches (C) have survived the transplantation procedure. The gap between the graft border (curved arrows) and the cheek pouch is now filled with inflammatory cells and loose erythrocytes. The cheek pouch (cp) lies just below the gap. $B$, cheek pouch blood vessel. Magnification: $\times 675$.

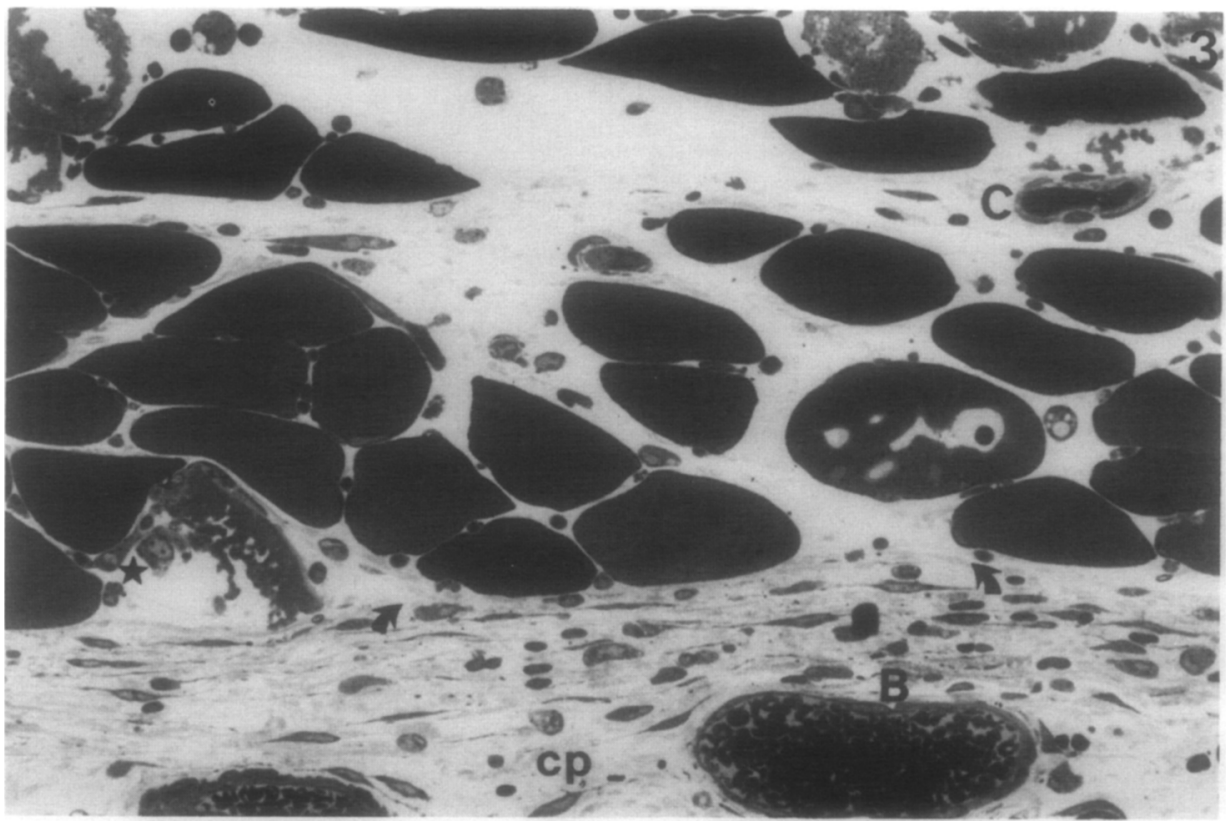




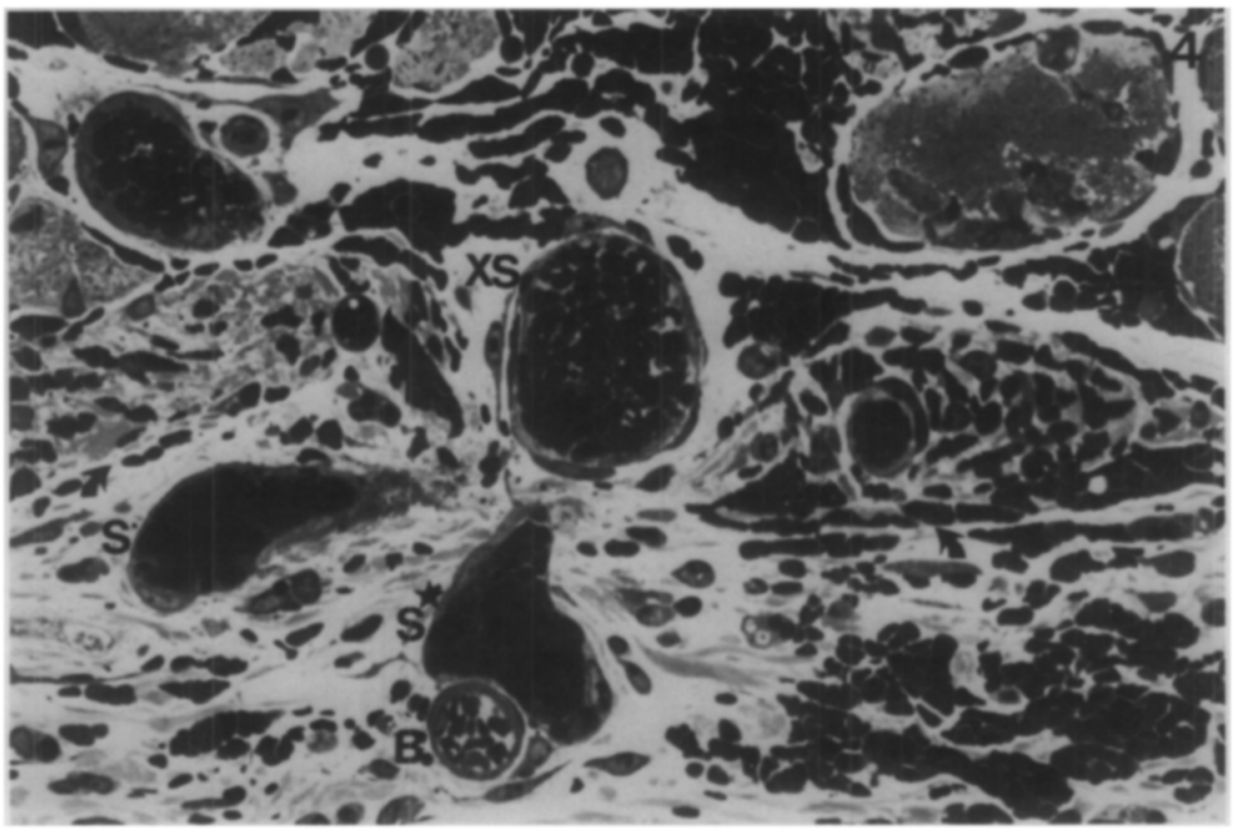

Fig. 4. This 2-day graft has a blood vessel seen in cross section (XS) which has grown sprouts (S). One sprout $\left(\mathrm{S}^{*}\right)$ has crossed the graft-cheek pouch border (curved arrows mark the bottom of the graft). The sprout, although located very close to a cheek pouch blood vessel (B), has not anastomosed with the cheek pouch vessel. The muscle fibers are in varying stages of degeneration. Magnification: $\times 585$.

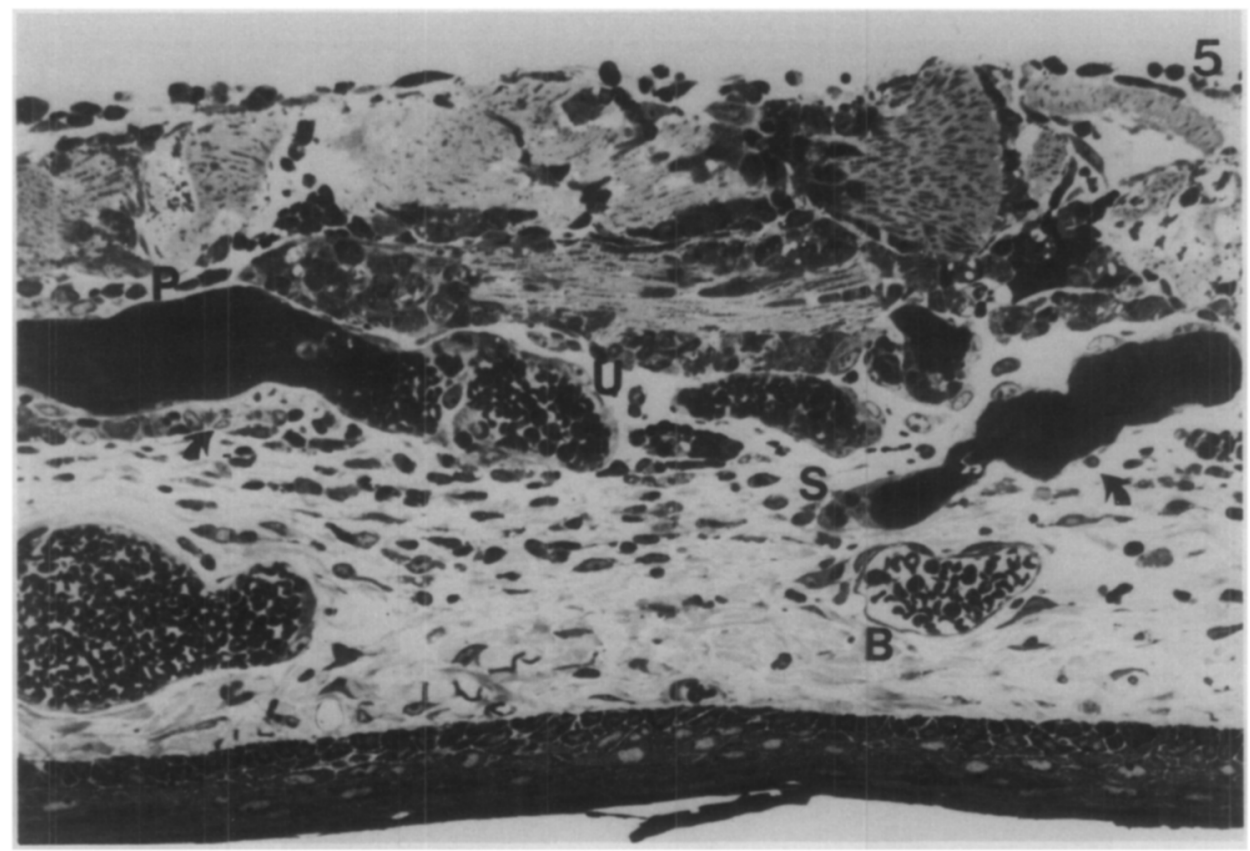




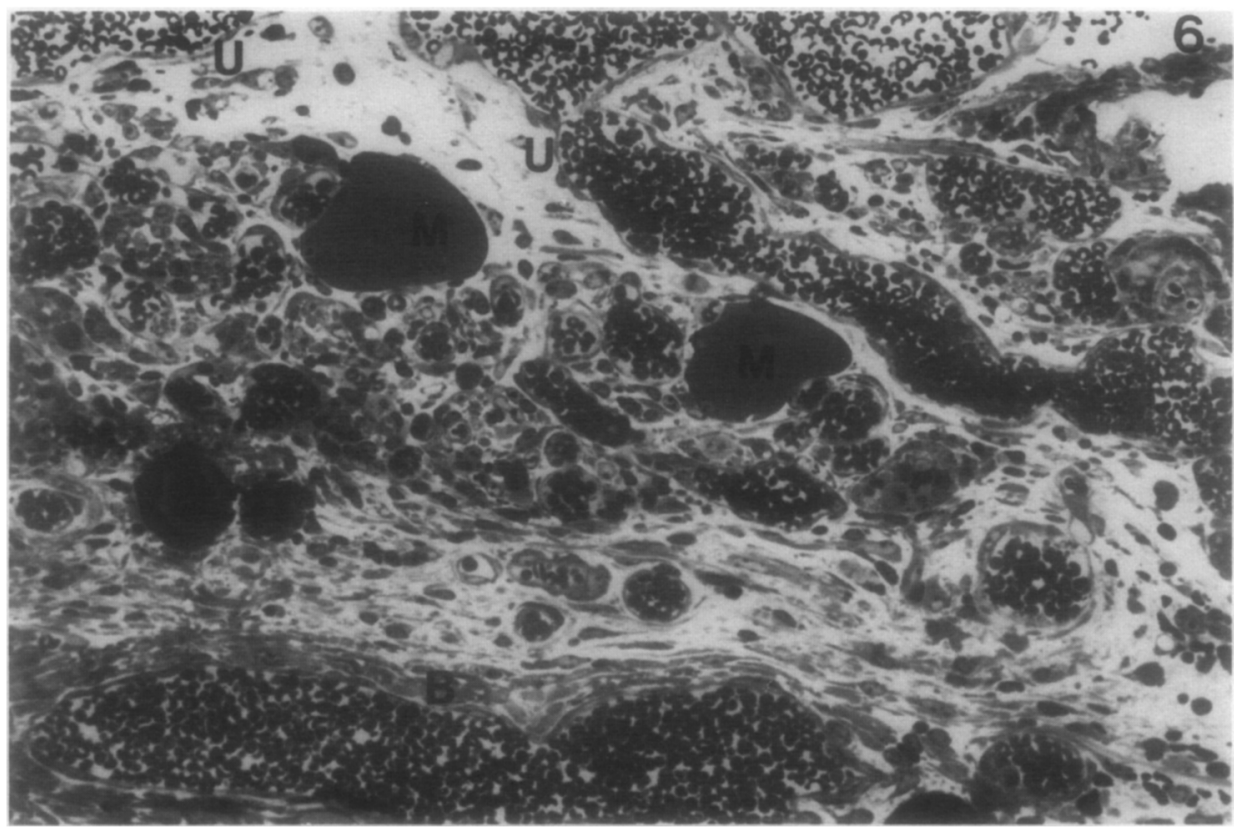

FIG. 6. Most muscle profiles are no longer recognizable at 3 days, although there are two muscle fibers (M) which seem relatively normal (see text). Most of the graft blood vessels (U) have unpacked erythrocytes. $B$, cheek pouch blood vessel. Magnification: $\times 380$.

unevenly thoughout the graft. The mean cross-sectional area of the myofibers was $466 \pm 54 \mu \mathrm{m}^{2}$.

By 14 days, muscle fibers had not increased significantly in cross-sectional area $\left(\bar{x}=403 \pm 48 \mu \mathrm{m}^{2}\right.$ ), but fibers were more numerous (Fig. 10). Compared to 5 days when from 5 to 10 fibers were observed in a cross section, there were 40 to 60 fibers. Because of different orientations, the number of fibers per cross section may not accurately reflect the actual number of fibers in a graft.

\section{Blood Vessels}

Intravital microscopy. No blood flow was observed in any vessel until 2.5 days after grafting. Between 2.5 and 3.5 days, flow in a large number of smalldiameter $(15-20 \mu \mathrm{m})$ vessels ranged from 0.02 to $0.06 \mathrm{nl} \cdot \mathrm{sec}^{-1}$ (Table 1). A large thin-walled vessel (Fig. 7) had a flow of $14.3 \mathrm{nl} \cdot \mathrm{sec}^{-1}$. By 4 days, all of the vessels in the graft demonstrated blood flow. The average diameter of vessels in which flow was measured increased but these data were not representative of the actual changes in blood vessels because flow was more easily measured in vessels near the upper surface of the graft. In vessels of comparable diameter

Fig. 5. This 2.5-day graft shows muscle fibers and graft blood vessels typical of this stage. One graft blood vessel has packed erythrocytes at one end (P) and well-scattered, unpacked erythrocytes at the other end (U). A graft blood vessel with packed erythrocytes along its entire length (S) has grown out of the graft (the bottom of the graft is marked by curved arrows) and is located near a cheek pouch blood vessel $(B)$. Magnification: $\times 380$. 


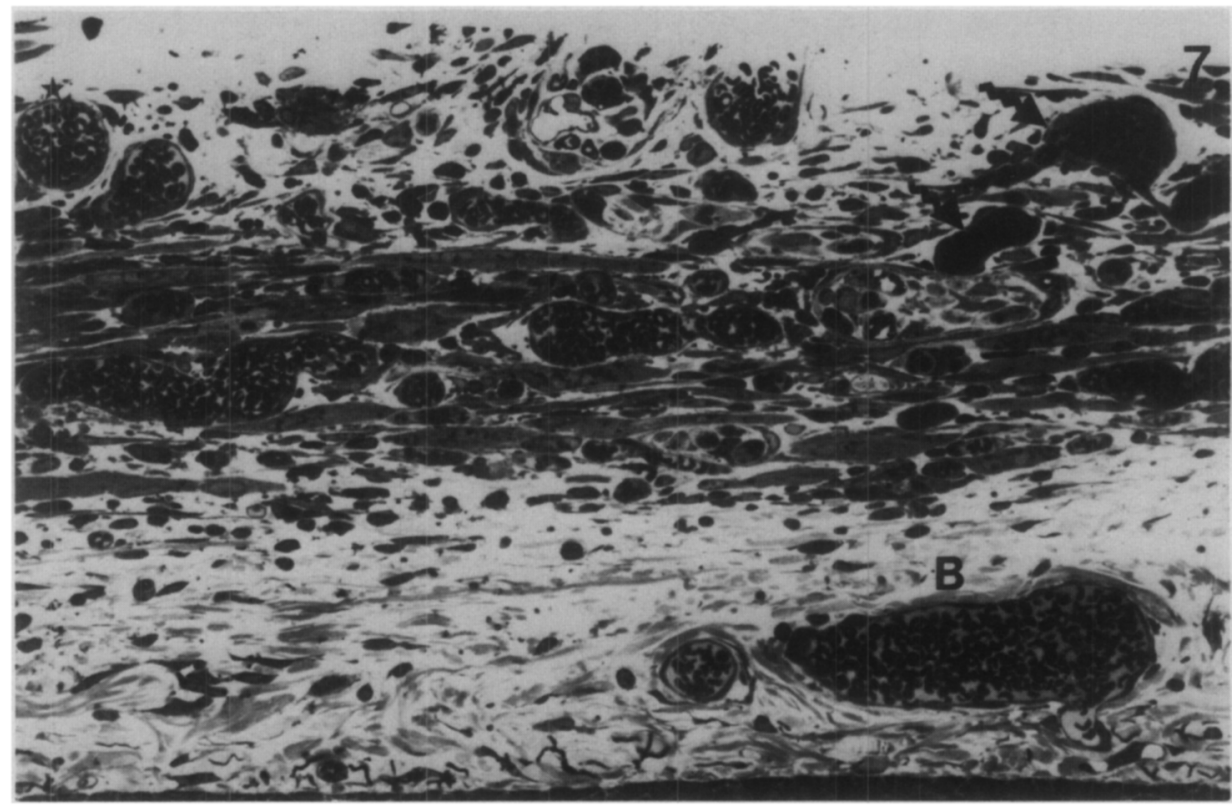

FIG. 7. Regenerating myotubes and large-diameter, thin-walled unpacked blood vessels are the most common features of 3.5-day grafts. A group of sinusoidal bulbs (wavy arrows) with packed erythrocytes is visible. The vessel marked with the star had a flow measured at $14.3 \mathrm{nl} \cdot \mathrm{sec}^{l}$. Magnification: $\times 340$

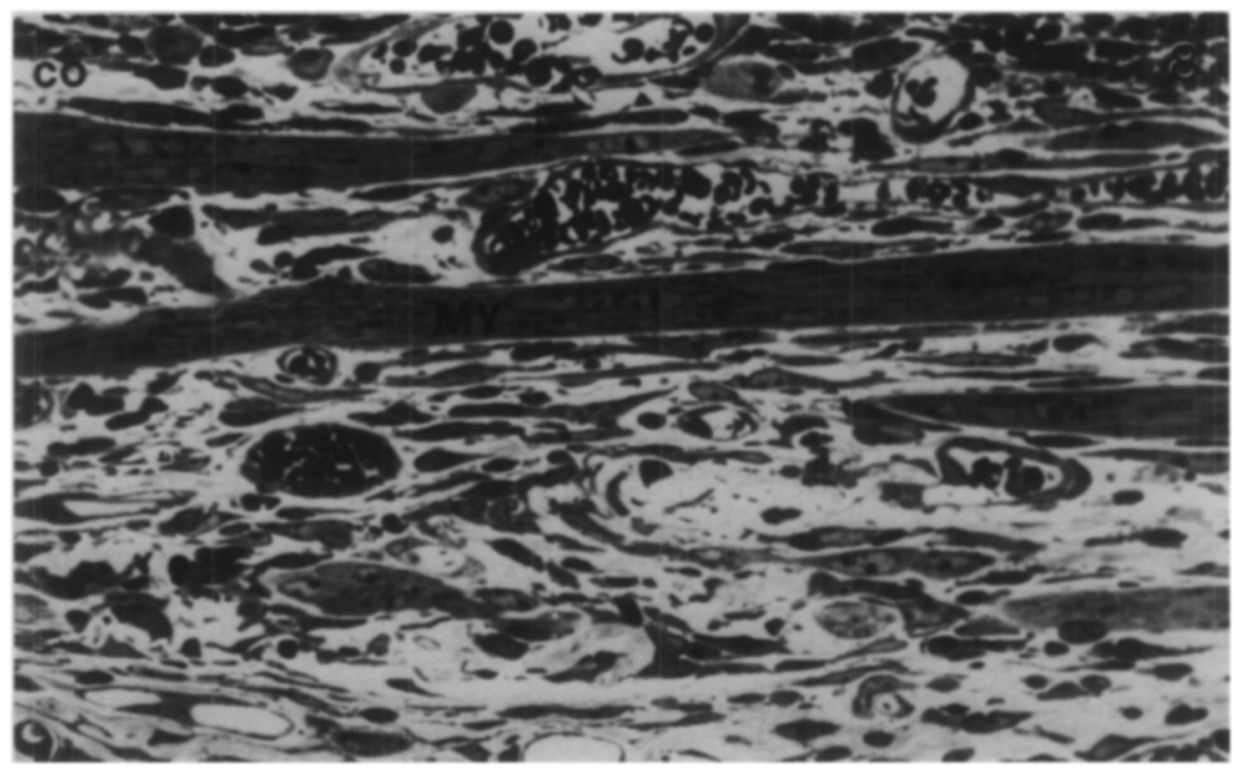

FIG. 8. Many myotubes (MY) are seen in this 4-day graft, but there is still a substantial amount of collagen (co). Magnification: $\times 850$. 


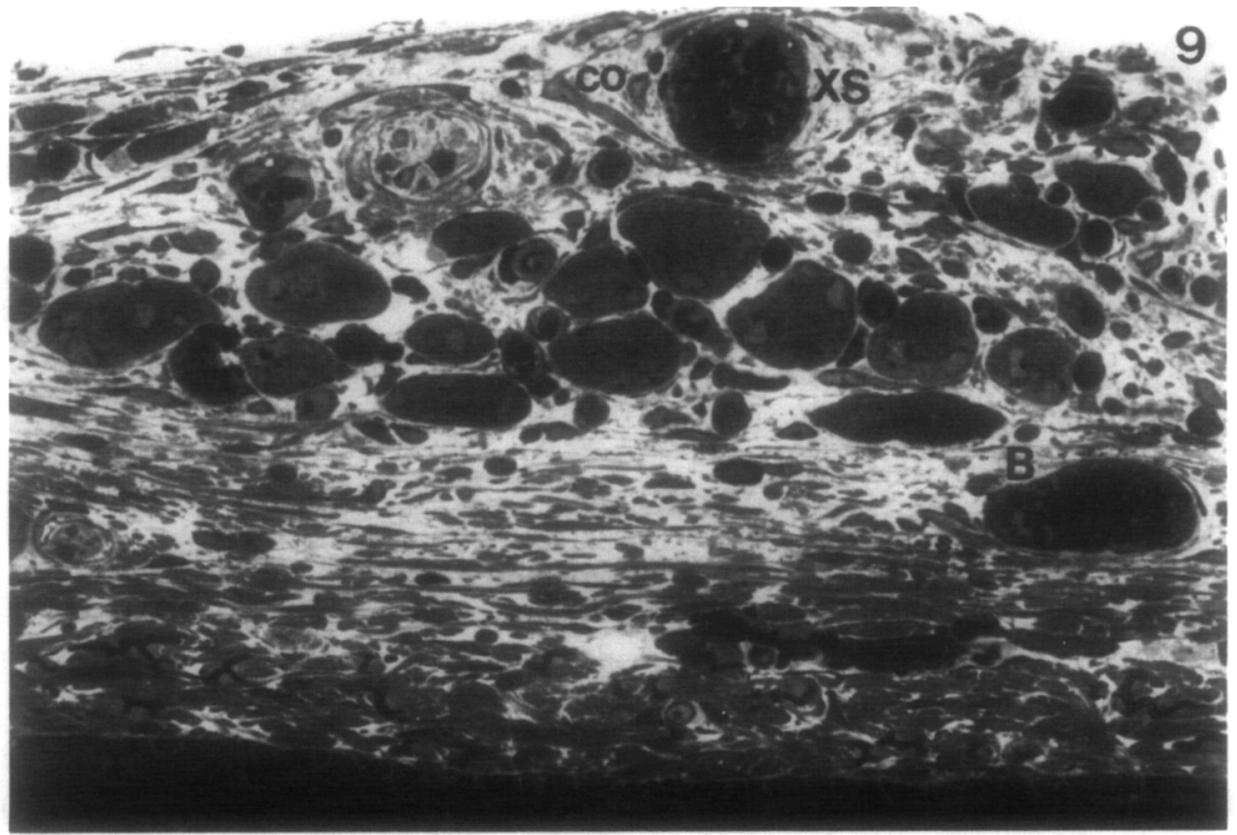

FIG. 9. In this 5-day graft, there are many myotubes and an occasional myofiber identified by the presence of peripheral nuclei. There is still a great deal of collagen (co). The blood vessels are quite small except for one seem in cross section (XS) which runs along the top of the graft and another $(B)$ which runs along the graft-cheek pouch border. Magnification: $\times 535$.

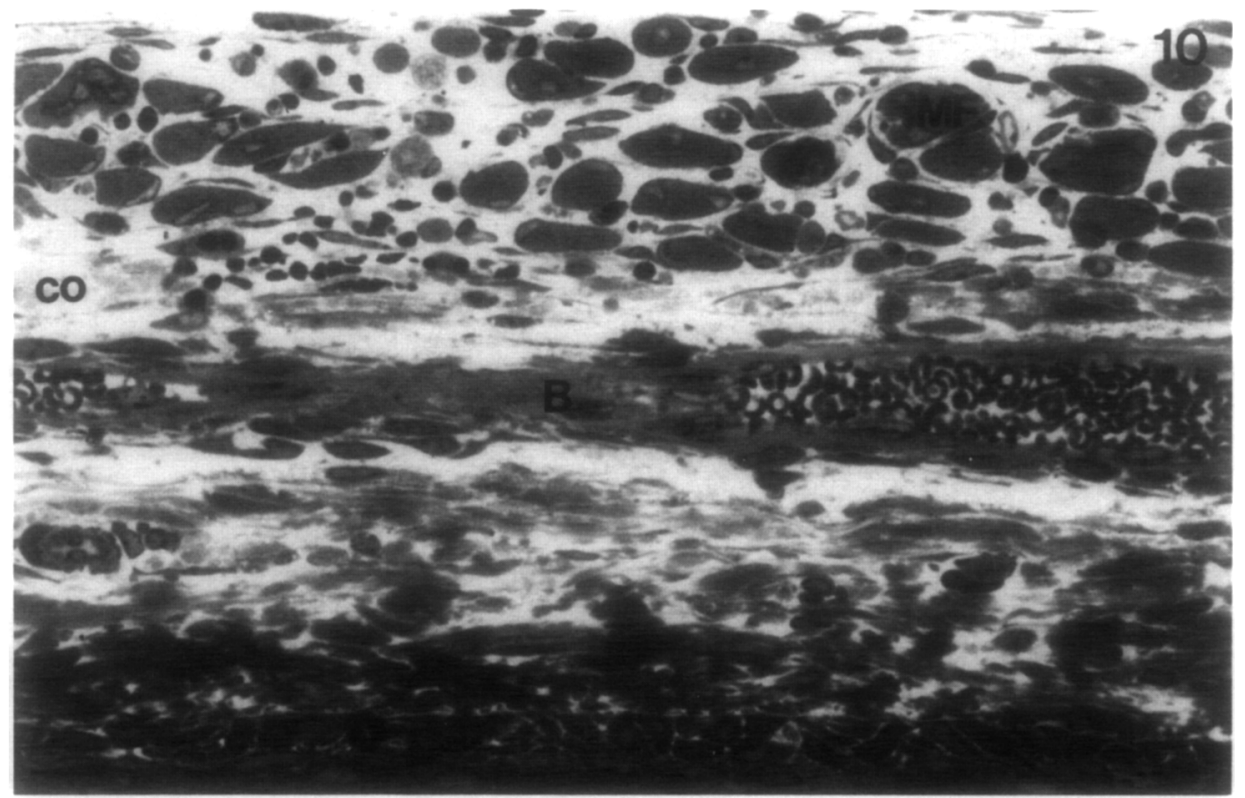

FIG. 10. Many myofibers (MF) are present and the amount of collagen (co) is greatly reduced by 14 days. Blood vessels in the graft are usually small-diameter and thin walled, but a large venule (B) can be seen at the graft-cheek pouch border. Magnification: $\times 565$. 
TABLE 1

Vessel Diameter and Resting Blood Flow of Blood Vessels in Control EdL Muscles, the Cheek Pouch, and in EDL Grafts in the Cheek Pouch

\begin{tabular}{lccc}
\hline & & $\begin{array}{c}\text { Vessel diameter } \\
(\mu \mathrm{m})\end{array}$ & $\begin{array}{c}\text { Blood flow } \\
\left(\mathrm{nl} \cdot \mathrm{sec}^{-1}\right)\end{array}$ \\
\hline $\begin{array}{l}\text { EDL } \\
\text { artery }\end{array}$ & & & \\
$\quad \begin{array}{l}\text { vein } \\
\text { Cheek pouch }\end{array}$ & $(N=6)$ & $22.8 \pm 1.0$ & $0.06 \pm 0.02$ \\
$\begin{array}{l}\text { Grafts } \\
2.5-3.5 \text { days }\end{array}$ & $(N=5)$ & $34.8 \pm 8.7$ & $0.15 \pm 0.04$ \\
& & $22.3 \pm 4.0$ & $0.48 \pm 0.28$ \\
$4-5$ days & $(N=7)$ & 108 & $0.06 \pm 0.01$ \\
14 days & $(N=1)$ & $28.0 \pm 2.9$ & 14.3 \\
\hline
\end{tabular}

Note. The mean vessel diameters are for those in which flow was measured. They are not necessarily representative of the graft. Blood flow was calculated from the equation: $\left.\dot{Q}(\mathrm{n}] \cdot \mathrm{sec}^{-1}\right)=$ [velocity $(\mathrm{mm} / \mathrm{sec}) / 1.6] \times\left[\Pi \operatorname{diameter}^{2}(\mu \mathrm{m}) / 4\right]($ LaLone and Johnson, 1978). The sample size $(N)$ gives the number of sites. Multiple readings of blood flow over a period of 2-4 min were made at a given site.

the rate of blood flow increased more than fivefold from 2.5 to 14 days. Even 14 days after grafting the blood flow in vessels of the graft was only $69 \%$ of the blood flow in vessels of comparable size in the cheek pouch and twofold greater than the blood flow in control EDL muscle.

Light microscopy. At 0.5 days, the regions surrounding the degenerating muscle fibers were infiltrated by loose erythrocytes, mast cells, and leukocytes (Fig. 2). Infiltration was most obvious at the border between the graft and the cheek pouch, especially in areas overlying cheek pouch blood vessels. Cheek pouch blood vessels were not affected at this stage except for the increase in leukocytes within the lumen and in the surrounding area.

Blood vessels in the graft were more like blood vessels in control EDL muscles (Fig. 1) than at any other subsequent stage. All the vessels observed had luminal diameters of less than $40 \mu \mathrm{m}$, and almost all the vessels observed had wellspaced erythrocytes having a normal shape (Fig. 2). Some capillaries, traced through serial sections, exhibited packing of erythrocytes in some segments with scattered erythrocytes in other segments. The capillaries, in general, were straight rather than sinusoidal.

Compared to control muscles the 0.5-day grafts had greatly decreased numbers of blood vessels. Grafts had fewer than 10 visible blood vessels, some single, but most in groups. These blood vessels were usually located near the sutures or in the center of the graft. The longest blood vessels were approximately 0.5 $\mathrm{mm}$ in length (Fig. 1).

The graft blood vessels by 1 day had undergone considerable change. Most of the vessels were small in diameter and thin walled (Fig. 2). The vessel wall consisted of endothelial cells and an occasional pericyte, without either connective tissue or smooth muscle. More obvious than the lack of differentiation of blood vessels was the packing of erythrocytes within them. Rather than being well scattered, they were located so close to each other that it was difficult to distinguish 
individual erythrocytes. In addition, erythrocytes were often spherical rather than biconcave. In contrast, cheek pouch vessels, although somewhat dilated, had well-spaced erythrocytes.

Most endothelial cells were normal, but some blood vessels had a morphologically abnormal outer layer with vacuoles. The endothelial cells at 1.5 days were swollen and had euchromatic nuclei. The swelling of the endothelial cells increased the thickness of blood vessel walls and was associated with a decreased diameter of the lumen. Many blood vessels had lumens barely large enough to accommodate one erythrocyte (Fig. 3). In addition, many blood vessels had cells (presumably endothelial cells) in the process of mitosis. The cells undergoing mitosis were most often found at the growing tip of the blood vessel.

The most striking feature at 2 days was the abundance of loose erythrocytes, both in the graft and the cheek pouch (Fig. 4). There were several blood vesscls in each graft, all but one with densely packed erythrocytes had crossed the graftcheek pouch border, but serial sections through entire graft segments never revealed them anastomosing with cheek pouch vessels despite their occasional close proximity to the latter (Fig. 4). Serial sections through to the end of one graft showed no connection between graft vessels and cheek pouch vessels. Graft blood vessels examined at $\times 600$ appeared to have endothelial cells in stages of active growth. Some were swollen, others were in the process of mitosis. Diapedesis of erythrocytes was observed in graft vessels that had crossed the graft-cheek pouch border and were located near a cheek pouch vessel.

One blood vessel in a 2-day graft was connected across the graft-cheek pouch border, i.e., serial sections showed a blood vessel in the cheek pouch, crossing the border, and in the graft. This vessel was easily distinguished from typical graft vessels as it had well-scattered erythrocytes along most of its length. It is unknown if this was a cheek pouch vessel which had grown into the graft, a graft vessel which had grown into the cheek pouch and anastomosed with a cheek pouch vessel, or a cheek pouch vessel which had grown into the graft and anastomosed with a graft vessel.

At 2.5 days, blood vessels in the cheek pouch had dilated such that their diameter was three to four times that of cheek pouch vessels at 1 day. Vessels in grafts at this stage were thin-walled endothelial tubes filled with densely packed erythrocytes. Their endothelial cells were typically enlarged but mitotic endothelial cells were observed less frequently. Some blood vessels had segments in which erythrocytes were well spaced (Fig. 5). The increased spacing of erythrocytes may result from an increased diameter of blood vessels or these blood vessels may have anastomosed to cheek pouch vessels at least at one end.

Thin-walled blood vessels in 3-day grafts showed evidence of budding and many short branches were present. This activity was most noticeable in vessels at the border between the graft and the cheek pouch. Mitoses were frequently seen in the walls. Blood flow was seen in some thin-walled vessels within the graft and this correlated histologically with the appearance of unpacked intravascular erythrocytes (Fig. 6). Also the appearance of blood flow could be correlated with the presence of connections between vessels in the cheek pouch and the graft. At this stage, and all subsequent stages, it became impossible to determine the origin (graft or host) of any particular vessel. 
Densely packed red blood cells were observed in graft vessels which had been shown, by intravital microscopy, to have no flow. In some cases these vessels opened into large sinusoid-shaped bulbs which were $100 \mu \mathrm{m}$ at their longest point. Serial sections showed these bulbs to be connected to circulation on one end only. These sinusoidal bulbs also contained densely packed erythrocytes. Other vessels exhibited normal spacing of erythrocytes in some segments but had packed erythrocytes in other segments. Serial sectioning showed areas of packed erythrocytes were usually found in the blind ends of blood vessel branches.

At 3.5 days, several blind-ended sinusoidal bulbs, densely packed with erythrocytes, were still seen. Blood vessels in the graft occupied at least $40 \%$ of the cross-sectional area. They were dilated, often 50-100 $\mu \mathrm{m}$ in diameter, and usually contained scattered erythrocytes. Connections between graft vessels containing scattered erythrocytes and cheek pouch vessels were common. The vessels, with their numerous branches, differed from control vessels in that they were sinusoidal rather than straight.

At 4 days, blood vessels were just as numerous as at 3.5 days, but vessels were smaller in diameter. The average blood vessel was less than $50 \mu \mathrm{m}$ in diameter. Vascular sprouting was still observed. Most of the blood vessels, both in the cheek pouch and the graft, were much less dilated and not as sinusoidal as at 4 days. All blood vessels had unpacked intravascular erythrocytes (Fig. 8).

The blood vessels at 14 days (Fig. 10) were still usually of the capillary type, the only evidence of large, differentiated vessels being those that filled the space between the graft and the cheek pouch. The vessels were straighter than at previous stages. The only large-diameter blood vessels were located either on the graft-cheek pouch border or along the top of the graft. A schematic representation of the sequence of events by which grafts in the cheek pouch are revascularized is presented in Fig. 11.

Fig. 11. Schematic drawings of the regeneration and revascularization of skeletal muscle bundles transplanted into the hamster cheek pouch. The time at which any given process occurred varied \pm 0.5 days between grafts. (A) 0 Day. Before transplantation the skeletal muscle bundle had an extensive, complex vascular system. At the time of transplantation, the blood flow was disrupted by the severing of all vascular connections. (B) 1.0 Day. The skeletal muscle fibers of the graft were vacuolated and some were infiltrated by phagocytic cells. Many blood vessels in the graft had degenerated, but a few had survived. Surviving blood vessels had packed erythrocytes. Veins in the cheek pouch had increased in diameter, but arteries remained unchanged. (C) 2.0 Days. The skeletal muscle fibers had undergone degeneration. Surviving blood vessels, which still contained packed erythrocytes, had growing sprouts. The sprouts grew linearly without branching. Some surviving blood vessel sprouts had grown from the graft into the cheek pouch. The cheek pouch veins had increased greatly in diameter, but neither veins nor arteries showed any signs of sprouting. (D) 2.5 Days. Some sprouts from surviving blood vessels had grown until they were in close proximity to blood vessels of the cheek pouch, but there was no evidence of anastomosis. (E) 3.0 Days. Muscle regeneration was at the fused myoblast stage. Graft and cheek pouch vessels had anastomosed resulting in reestablishment of circulation in the graft. Graft blood vessels with flow were large and branched. Flow in some graft blood vessels alternated direction. Dead-end sinusoidal bulbs presumably resulted when blood vessels were anastomosed with vessels in the cheek pouch circulation at only one end. (F) 4 Days. Myotubes were common. Blood vessels in the grafts were smaller than at 3.0 days. The complete circulatory system which was reestablished in the graft was not as complex as the circulatory system of control EDL muscles. The blood vessels of the cheek pouch had decreased to control size. 
A. O DAYS

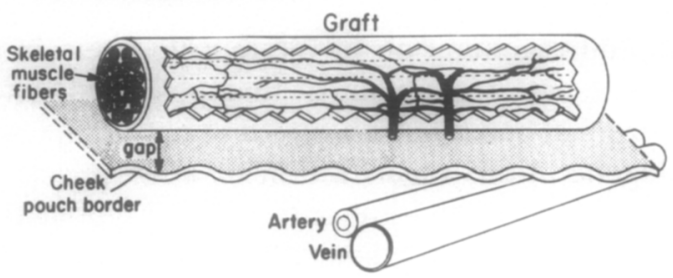

B. 1.0 DAYS

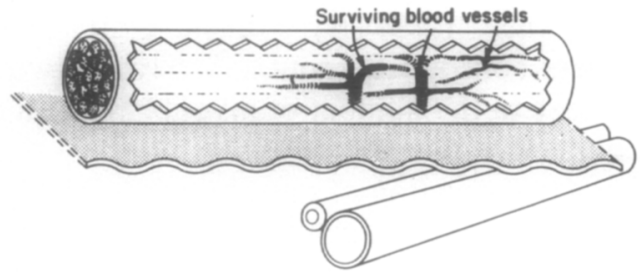

C. 2.0 DAYS

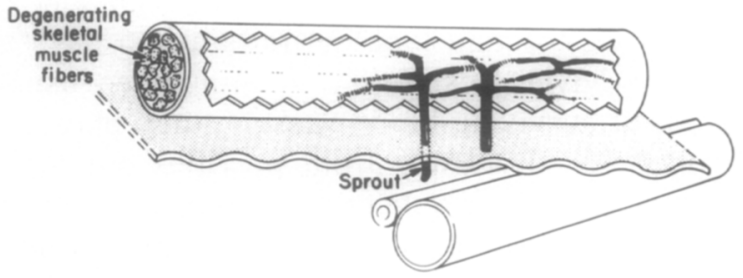

D. 2.5 DAYS

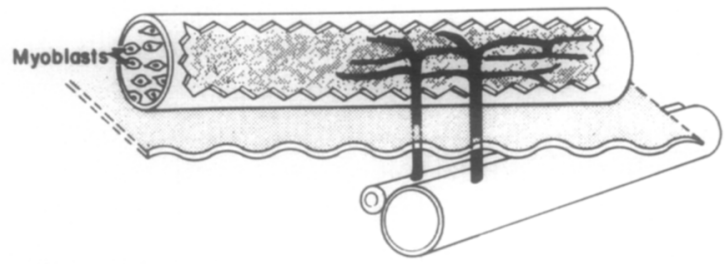

E. 3.0 DAYS

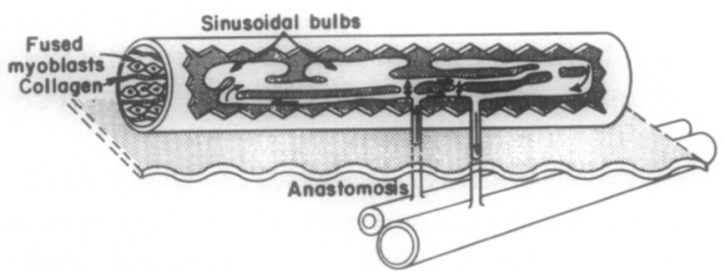

F. 4.0 DAYS

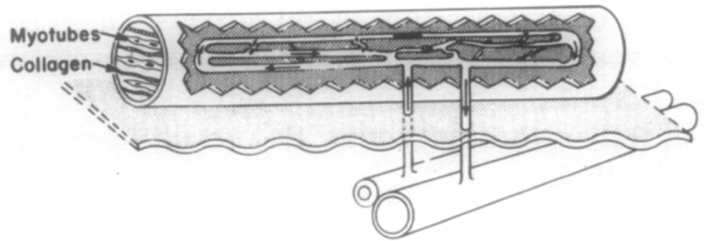




\section{DISCUSSION}

The degeneration and regeneration of the muscle fibers in a graft located in a cheek pouch follows a pattern similar to that observed when the entire EDL was grafted into its original bed (Hansen-Smith et al., 1980; Carlson and Faulkner, 1983). Following degeneration, regeneration of new muscle fibers occurs, presumably from satellite cells which differentiate into myoblasts (Snow, 1977). The myoblasts fuse to form myotubes and then differentiate into myofibers. Regeneration of skeletal muscle fibers in the cheek pouch of the hamster follows a similar time course to that of fibers regenerating in $100-\mathrm{mg}$ orthotopic EDL grafts in rats (Carlson and Faulkner, 1983). By 5 days, grafts in the cheek pouch had myofibers and the fibers contracted spontaneously. Between 5 and 14 days, the changes were an increase in the number of myofibers and a concomitant decrease in the amount of connective tissue.

In contrast, the revascularization of muscle transplanted into the cheek pouch differed significantly from the revascularization of orthotopically transplanted EDL muscles (Hansen-Smith et al., 1980). This study demonstrates that some of the graft blood vessels survive and elongate. As blood vessels grow at a rate of less than $0.5 \mathrm{~mm} /$ day (Clark and Clark 1939; Eriksson and Zarem, 1980), the presence of blood vessels $0.5 \mathrm{~mm}$ long in grafts at 0.5 days after grafting can only be explained by survival. Despite the small number, usually about 10 , and size, $0.5 \mathrm{~mm}$ or less, of surviving blood vessels, they play a major role in the revascularization of grafts. Evidence from previous studies of whole-muscle grafts has favored the interpretation that revascularization occurs primarily from the ingrowth of vessels located outside the graft (Hansen-Smith et al., 1980). In contrast, grafts in the hamster cheek pouch revascularize when surviving blood vessels grow from the graft into the cheek pouch and anastomose with cheek pouch vessels. Sprouts were never observed growing from cheek pouch vessels into the graft. At 1.5 days, mitosis of endothelial cells of graft vessels was common, whereas mitoses in cheek pouch vessels were rare. Compared to the passive role of the blood vessels in the cheek pouch, we conclude that the blood vessels in the graft play an active role in revascularization.

Surviving blood vessels may be unique to this particular system because of the status of the cheek pouch (Shepro et al., 1963), the small mass of the graft (Carlson and Faulkner, 1983), the proximity of the cheek pouch vessels, or some combination of these factors. Blood vessels in larger muscle grafts may not survive. Alternatively, because of their scarcity, surviving vessels in large muscle grafts may be missed unless serial sectioning is used. Serial sectioning is impractical in whole muscles such as the rat EDL.

The packing of erythrocytes in graft vessels was not likely a fixation artifact because the cheek pouch vessels in the same sections appeared normal. Erythrocyte packing in graft vessels between 1 and 2.5 days could result from a decreased lumen cross-sectional area. Swelling of endothelial cells decreased the size of the vessel lumen between 0.5 and 1 day. A decreased vessel lumen is also associated with a loss of plasma from blood vessels which is common in inflammatory reactions (Zweifach, 1973). In grafts, blood vessels are isolated from the circulation and the loss of plasma cannot be replaced. The spherical, rather than biconcave shape of the erythrocytes may also result from ischemia and/or tissue damage (Brånemark, 1968). 
More difficult to explain is the dense erythrocyte packing observed at 2 to 2.5 days when the blood vessels are elongating. Their packing may reflect the movement of erythrocytes into a blind-ended tube. The sinusoids, packed with erythrocytes, observed at 3 to 3.5 days may result as vessels which are blind-ended at one end and anastomose with the cheek pouch circulation at the other end. As surviving blood vessels are often seen in groups, rather than singly, these blood vessels may be connected to each other. Serial sections suggest that many of them are connected and consequently one graft blood vessel anastomosing to a cheek pouch blood vessel would result in erythrocyte packing in several graft vessels, since they would all be blind-ended. Since no sinusoids with packed erythrocytes were seen at 4 days, previously blind ends must be joined to the circulation to establish flow.

The large number of loose erythrocytes seen at 2 to 3 days may result from the rupture of some blind-ended vessels. New blood vessels are more fragile than older ones (Schoefl, 1963) and the increase in pressure as they are joined to the circulation may rupture some of them. Rupturing of graft vessels during transillumination for intravital microscopy occurred on two occasions. These grafts were not included in the study.

For EDL grafts in cats, White et al. (1981) reported that as long as 280 days after transplantation resting blood flows expressed relative to tissue mass were fourfold higher than the values for control EDL muscles. From 2.5 days when flow was first observed to 14 days, blood flow in vessels of similar size increased more than fivefold. This increase resulted in blood flows in grafts which were twofold greater than the flows in vessels of comparable size in control EDL muscles. The proliferation of vessels 3 to 4 days after grafting resulted in almost half the cross section of the graft being composed of blood vessels. The combination of increased resting flow per unit cross-sectional area and the increased crosssectional area of vessels resulted in resting flows in grafts in the cheek pouch significantly greater than control EDL muscles. The area of vessels regressed rapidly back toward control values. If such a proliferation of blood vessels occurs in whole 3-g EDL muscles of cats, it is masked by the peripheral to central gradient of regenerative events (Carlson and Faulkner, 1983). The peripheral to central gradient of regenerative events results in vascular proliferation in the central area after the periphery has already undergone regression. The mechanism by which resting blood flow in whole-muscle grafts is increased remains unclcar.

The survival of endothelial cells for several days in the ischemic graft led us to reject our first hypothesis that all of the graft blood vessels would degenerate. We rejected the hypothesis that blood vessels grew into the graft on the basis of the passive role of blood vessels in the cheek pouch and the active growth of sprouts from surviving vessels in the graft. Skeletal muscle fibers from the EDL muscle regenerated successfully in the cheek pouch during the 14 days of observation.

\section{ACKNOWLEDGMENTS}

William L. Joyner, Department of Physiology and Biophysics, University of Nebraska College of Medicine, Omaha, was most generous in his assistance to us in the development of the technique for transplantation of skeletal muscle into the hamster cheek pouch. Christopher Watters aided with the transplantation of muscle and Kevin K. McCully assisted in the collection of data on blood flow. 
The research was supported by a Program Project from United States Public Health Service Grant NS-17017 from the National Institute of Neurology, Communicative Diseases and Stroke, National Institutes of Health, and the Michigan Heart Association.

\section{REFERENCES}

BrÅnemark, P-I. (1968). Rheological aspects of low flow states. In "Microcirculation as Related to Shock" (T. Shepro and G. P. Fulton, eds.), pp. 161-180. Academic Press, New York.

Carlson, B. M. (1976). A quantitative study of muscle fiber survival and regeneration in normal, predenervated, and Marcaine-treated free muscle grafts in the rat. Exp. Neurol. 52, 42I-432.

Carlson, B. M., AND Faulkner, J. A. (1983). The regeneration of skeletal muscle fibers following injury. Med. Sci. Sports Exerc., 15, 187-198.

Carlson, B. M., and Gutmann, E. (1975). Regeneration in grafts of normal and denervated rat muscles: contractile properties. Pfluegers Arch. 353, 215-225.

Clark, W., LeGros, E., and Blomfield, L. B. (1945). The efficiency of intramuscular anastomoses with observations on the regeneration of devascularized muscle. J. Anat. 79, 15-32.

Clark, E. R., AND ClaRK, E. L. (1939). Microscopic observations on the growth of blood capillaries in the living mammal. Amer. J. Anat. 64, 251-301.

CLOSE, R. (1965). The relation between intrinsic speed of shortening and duration of the active state of muscle. J. Physiol. (London) 180, 542-559.

Cornish, K. G., Joyner, W. L., and Girmorf, J. P. (1979). Direct evidence for the presence of a different converting enzyme in the hamster cheek pouch. Circ. Res. 44, 540-544.

ERIKsson, E., AND Zarem, H. A. (1980), Growth and differentiation of blood vessels. In "Microcirculation" (G. Kaley and B. M. Altura, eds.), Vol. 1, pp. 393-419. Univ. Park Press, Baltimore.

Faulkner, J. A., Niemeyer, J. H., Maxwell, L. C., and White, T. P. (1980). Contractile properties of transplanted extensor digitorum longus muscles of cats. Amer. J. Physiol. 238, C120-C126.

Greendlatt, M., AND ShubiK, P. (1968). Tumor angiogenesis: transfilter diffusion studies in the hamster by the transparent chamber technique. J. Nat. Cancer Inst. 41, 111-124.

Greenblatt, M., Kaufman, J., and Choudari, K. U. R. (1971). Functioning heart homografts in hamsters. Transplantation 11, 50-55.

Hansen-Smith, F. M., Carlson, B. M., and IRwin, K. L. (1980). Revascularization of the freely grafted extensor digitorum longus muscle in the rat. Amer. J. Anat. 158, 65-82.

Hurley, J. V., AND Edwards, B. (1969). Acute inflammation: A combined light- and electronmicroscope study of the vascular response to incisional and crushing injury of skeletal muscle in the rat. J. Pathol. 98, 41-52.

LALONE, B. J., AND Johnson, P. C. (1978). Estimation of arteriolar volume flow from dual-slit red cell velocity: an in vivo study. Microvasc. Res. 16, 327-339.

Markley, J. M., Faulkner, J. A., AND Carlson, B. M. (1978). Regeneration following transplantation of skeletal muscles in monkeys. Plast. Reconstr. Surg. 62, 415-422.

Mong, F. S. F. (1977). Histological and histochemical studies on the nervous influence on minced muscle regeneration of triceps surae of the rat. J. Morphol. 131, 451-462.

SCHOEFL, G. I. (1963). Studies on inflammation. III. Growing capillaries: Their structure and permeability. Virchows Arch. Pathol. Anat. 337, 97-141.

Shepro, D., Kula, N., and Hatkftt, I. A. E. (1963). The role of the cheek pouch in effecting transplantation immunity in the hamster. J. Exper. Med. 117, 749-754.

SNow, M. H. (1977). Myogenic cell formation in regenerating rat skeletal muscle injured by mincing. Anat. Rec. 188, 181-199.

VRACKo, R., AND Benditt, E. P. (1972). Basal lamina: The scaffold for orderly cell replacement. $J$. Cell Biol. 55, 406-419.

White, T. P., Maxwell, L. C., Sosin, D. M., and Faulkner, J. A. (1981). Capillarity and blood flow of transplanted skeletal muscles of cats. Amer. J. Physiol. 241, H630-H636.

ZWEIFACH, B. W. (1973). Microvascular aspects of tissue injury. In "The Inflammatory Process" (L. Grant and R. T. McCluskey, eds.), Vol. 2, pp. 3-46. Academic Press, New York. 